\title{
Disminución de la incidencia de defectos de cierre del tubo neural en el Hospital Universitario de Neiva: posible efecto de la promoción del consumo de ácido fólico
}

\author{
Henry Ostos ${ }^{1}$, Gilberto Astaiza ${ }^{1}$, Francisco García ${ }^{2}$, Miller Bautista ${ }^{3}$, Favio Rojas ${ }^{1}$, Antonio Bermúdez ${ }^{4}$ \\ ${ }^{1}$ Facultad de Salud, Universidad Surcolombiana, Neiva, Huila, Colombia \\ ${ }^{2}$ Secretaría de Salud Departamental del Huila, Neiva, Colombia \\ ${ }^{3}$ Hospital San Vicente de Paúl, Garzón, Huila, Colombia \\ ${ }^{4}$ Laboratorio Nacional de Referencia, Instituto Nacional de Salud, Santa Fe de Bogotá, D.C., Colombia
}

\section{Resumen}

Los defectos del tubo neural (DTN) son defectos de nacimiento, del cerebro o la médula espinal, los cuales llevan a la muerte o a la discapacidad. Las causas de los DTN son desconocidas. Se piensa que ocurren por una interacción de factores genéticos y ambientales. En 1997, se observó un significativo aumento en la incidencia de defectos del tubo neural en el Hospital Universitario de Neiva $(p=0,035)$, respecto al año anterior. El primer esfuerzo de control consistió en promover el consumo de ácido fólico siguiendo las recomendaciones universales de suplementación y fortificación (CDC Folic Acid Resource Guide). En este estudio se evaluó la incidencia de los DTN durante 1998, como posible indicador del impacto de las medidas tomadas. Se identificaron los nacidos con DTN, los nacidos con síndrome de Down o con labio o paladar hendido, la proporción de abortos y la de nacidos muertos. La tasa de DTN disminuyó en forma significativa $(p=0,024)$ respecto a 1997 y su diferencia con la tasa de Latinoamérica no fue significativa $(p=0,526)$. Se concluyó que la incidencia fue menor en 1998 y regresó a la tasá esperada para la región. Aunque se desconoce la causa del descenso, es posible que sea el resultado de la divulgación de los beneficios de una buena nutrición materna, la suplementación pregestacional con ácido fólico, la fortificación de la harina de trigo, o una sumatoria de todas las medidas. Se recomienda desarrollar un estudio de actitudes y prácticas sobre el uso de ácido fólico en la población de mujeres de Neiva en edad fértil y establecer un protocolo de vigilancia activa de los DTN.

Palabras clave: congénito, genético, fólico, neural, prevención.

Low incidence of congenital neural tube defects at the Neiva University Hospital: possible effect of folic acid supplementation

\begin{abstract}
Neural tube defects (NTD) are brain or spinal cord congenital defects, causing death or severe disability. Causes of NTDs are unknown. It seems that NTDs occur due to environmental and genetic interactions. In 1997, a significant increase in the rate of NTDs $(p=0.035)$ related to 1996 was observed in the maternity wing at the University Hospital in Neiva, Colombia. Health authorities initiated a program according to the universal recommendation about folic acid supplementation and fortification to prevent NTDs (CDC Folic Acid Resource Guide). The aim of this study was to measure the incidence of NTDs in 1998, related to previous years, as a possible way to measure the impact of the actions adopted by the community and health authorities. The incidence of NTDs, Down syndrome, cleft
\end{abstract}


or palate lip, stillbirth and fetal losses, was measured for 1998. The rate of NTDs was similar compared to that of Latin America $(p=0.526)$, but significantly less than that for $1997(p=0.024)$, and reached similar values than those of $1996(p=0.807)$. The explanation for this reduction of NTDs is not clear now, but we conclude that there is an apparent impact of advice on preconceptional folic acid supplementation together with improvement in dietary intake through grain and flour fortification. We recommend to evaluate the attitudes and practices of childbearing women regarding the benefits of folic acid before conception and during pregnancy. It is also important to continue the surveillance program for NTDs.

Key words: congenital, genetic, folate, neural, prevention.

La médula espinal y el cerebro se desarrollan a partir de una tira de células que corren a lo largo del dorso del embrión. Dos a tres semanas después de la concepción, aparece una grieta en el centro de lo que será la espalda del bebé. Gradualmente, la grieta se profundiza y los extremos de la tira se curvan, acercándose entre sí y fusionándose para formar el tubo neural. El tubo neural se transforma más tarde en médula espinal y cerebro (1).

En los casos con defecto del tubo neural (DTN), el tubo falla en cerrarse apropiadamente y ocurre el defecto en el cierre o la lesión. Son defectos de nacimiento, del cerebro o la médula espinal, los cuales usualmente llevan a la muerte o a la discapacidad. Casi siempre, las meninges o el LCR protruyen a través de la apertura.

Los defectos más comunes del tubo neural son la espina bífida en el $60 \%$ de los casos, anencefalia en $35 \%$ y encefalocele en $5 \%$ (2). Las anomalías del cordón espinal pueden resultar en parálisis motora, deformidades del esqueleto, pérdida sensorial e incontinencia intestinal y urinaria. Los bebés que nacen con anencefalia son mortinatos o sufren muerte neonatal temprana. El encefalocele es usualmente fatal, pero la supervivencia es posible con pequeñas lesiones y cirugía temprana. La malformación puede resultar en compromisos sensoriales, cognitivos y motores.

Las causas de los DTN son desconocidas. Se piensa que ocurren por una interacción de factores genéticos y ambientales. Algunos factores

Correspondencia: Henry Ostos, Facultad de Salud, Universidad Surcolombiana, Neiva, Huila, Colombia

Recibido: 5/11/99; aceptado: 23/02/00 asociados con un riesgo incrementado de los DTN incluyen embarazo previo con DTN (3), mujer con un pariente cercano que haya tenido DTN, raza o etnia, ya que es más común en mujeres blancas y en hispánicas (4), diabetes materna insulinodependiente, uso materno de medicación anticonvulsa con ácido valproico o carbamazepina, nivel socioeconómico y educativo bajo, obesidad diagnosticada por médico y exposición prolongada a temperaturas altas en los primeros meses del embarazo.

Las consecuencias de estos defectos son la discapacidad física de por vida en los casos menos severos o en los que sobreviven a las cirugías. La tasa de mortalidad es elevada en el periodo neonatal temprano para los casos severos y todas las anencefalias. Los costos sociales son muy altos y, en Estados Unidos, el impacto ecómico se ha calculado en U.S.\$200 millones por año para el cuidado médico y los costos quirúrgicos de los niños con DTN (5).

La principal estrategia para la prevención es la promoción de un estilo de vida saludable en la mujer en edad reproductiva, con el mejoramiento en su nutrición, la cual debe contener un mínimo de ácido fólico de $400 \mu \mathrm{g} / \mathrm{día}$, y suplementación preconcepcional enfocada hacia las mujeres que estén en edad fértil y tengan probabilidad de quedar embarazadas (6). El ácido fólico no previene la totalidad de los DTN, pero sí los previene en 50 a $70 \%$ de los casos (7). El mecanismo por el cual se logra este efecto está en discusión y los estudios se enfocan en el gen de la tetrahidrofolatoreductasa y la vía metabólica de los folatos (8).

Algunas razones por las cuales falla la estrategia son las actitudes y prácticas de la mujer. En un 
informe descriptivo realizado en las Islas Británicas (9), se plantea un interrogante a la efectividad de las políticas sobre ácido fólico, porque no se observó disminución de los DTN después de adoptar las normas de suplementación. Aunque las cifras del estudio sólo se mencionan superficialmente, es importante el análisis resultante. Se plantean, entre otras posibles causas del fracaso, que las mujeres no planifican el embarazo, o lo hacen, pero su actitud frente a la propuesta de utilizar el ácido fólico es negativa, o no tienen seguridad de los beneficios de adoptar las recomendaciones.

En 1996 y 1997 se observó un incremento en la incidencia de los DTN en el Hospital Universitario de Neiva (10). En 1997, la tasa fue superior a la informada para los años anteriores en ese hospital y superior a la de Irlanda, que ha tenido las tasas de prevalencia más altas del mundo (11).

Desde que se observó el incremento en los DTN, el Departamento de Genética comenzó la motivación del personal médico y paramédico para incentivar el consumo de ácido fólico entre las mujeres en edad fértil. Pero no fue sino hasta 1997 que se alertó sobre un aumento aún mayor y, entonces, se iniciaron actividades de intervención desde la Secretaría de Salud Departamental con el apoyo del Instituto Nacional de Salud y del Departamento de Genética de la Universidad Surcolombiana. El primer esfuerzo consistió en promover el consumo de ácido fólico siguiendo las recomendaciones de los Centros de Control de Enfermedades (CDC) $(1,12)$.

Las medidas consistieron en campañas de divulgación de los beneficios de un consumo elevado de alimentos con alto contenido de folatos a través de medios masivos de comunicación como las radiodifusoras de la ciudad. Se hicieron panfletos y volantes con información sobre cómo mejorar la nutrición familiar y boletines con dibujos explícitos, dirigidos al público en general, sobre el origen de los DTN y la forma de prevenirlos en un alto porcentaje de casos. En el hospital, se dictaron conferencias para insistir -entre el personal médico y paramédico- en la necesidad de crear estrategias para motivar a las mujeres en edad fértil a consumir folatos antes de quedar en embarazo. En 1998, gracias a una resolución de la gobernación departamental, se hizo efectiva la norma sobre la fortificación de harinas con ácido fólico, decretada dos años antes por el gobierno nacional $(4,13)$.

El propósito del presente estudio es comparar la incidencia de las anomalías del cierre del tubo neural en el Hospital Universitario de Neiva durante 1998 con la de años anteriores, con el fin de establecer un posible indicador del impacto de las medidas de intervención adoptadas desde 1996 y como punto de partida para evaluar la eventual fortificación de la harina de trigo con ácido fólico.

\section{Metodología}

Se propuso identificar los nacidos con DTN. Para esto, se consultaron los libros de registro de nacimientos en sala de partos, los egresos, las historias clínicas de los casos detectados y las estadísticas vitales por meses. Los datos correspondientes a 1998 se buscaron en el Hospital General de Neiva, hoy Hospital Universitario. También se buscaron los nacidos con síndrome de Down o con labio o paladar hendido, como fenotipos centinela de patología genética en la misma maternidad. Como indicador de mortalidad fetal, se buscó la proporción de abortos y la de nacidos muertos.

Con el propósito de tener cifras de base para la comparación con otras maternidades, se recogió la misma información en la Clínica Federico Lleras del Seguro Social en Neiva y en dos poblaciones cercanas, Baraya y Palermo, en hospitales de nivel I que remiten los embarazos complicados a Neiva. Así mismo, se decidió buscar información en otro hospital que no remitiera a Neiva y se escogió Garzón, que tiene un hospital de nivel II (cuadro 1).

La información se tabuló para establecer las tasas de incidencia de defectos de cierre del tubo neural, de sindrome de Down, las razones de mortinatos y las de abortos. Se compararon con la prueba estadística $\chi^{2}$ a un nivel de significancia de $p<0,05$.

\section{Resultados}

En el Hospital Universitario de Neiva hubo 7 defectos de tubo neural de sexo femenino y 1 de 
Cuadro 1. Instituciones visitadas para indagar sobre defectos de tubo neural, por municipio.

\begin{tabular}{lll}
\hline Municipio & \multicolumn{1}{c}{ Institución } & \multicolumn{1}{c}{ Fuente } \\
\hline Neiva & Hospital Universitario & $\begin{array}{l}\text { Libro de sala de partos } \\
\text { Estadística } \\
\end{array}$ \\
& ISS, Clínica Federico Lleras Acosta & Libro de sala de partos \\
& & Libro de ingresos y egresos de la UCl \\
Palermo & Hospital San Francisco de Asis & Registros de estadistica \\
Baraya & Hospital Tulia Durán de Borrero & Estadística \\
Garzón & Hospital San Vicente de Paúl & Indicadores de gerencia \\
& & Libro de ingresos de pediatría
\end{tabular}

sexo masculino. Otros 6 niños nacieron con síndrome de Down, 1 con labio hendido y 2 con paladar hendido (cuadro 2). La distribución por fechas de nacimiento es homogénea a partir del segundo trimestre (figura 1).

En los hospitales de Baraya y Palermo, no hubo casos de defectos congénitos ni nacidos muertos. En Garzón, en el Hospital San Vicente de Paúl, hubo 3 defectos de tubo neural y un síndrome de Down (cuadro 3).

En la Clínica Federico Lleras del Seguro Social en Neiva, no cuentan con registros en sala de partos para los diagnósticos de anomalías congénitas. Por tanto, sólo se registran los bebés que sean hospitalizados en la unidad de cuidado intensivo y no se registran los datos de los que son dados de alta sin complicaciones. Además, las historias clínicas están en manos de los pacientes y no se pueden consultar para verificar los diagnósticos. En esta maternidad se registraron 3 defectos de tubo neural y 2 de síndrome de Down (cuadro 4).

\section{Resultados}

En el Hospital Universitario, la tasa de defectos de tubo neural para 1998 permanece mayor que la informada para Latinoamérica, pero la diferencia no es significativa $\left(\chi^{2}=2,3 ; p=0,129\right)$ (cuadro 5). La comparación de la tasa de los DTN de 1998 con la tasa encontrada en el estudio de 1997 (cuadro 6) muestra una disminución significativa $\left(\chi^{2}=5,06 ; p=0,024\right)$, pero, comparada con la de 1996 la diferencia no es significativa $\left(\chi^{2}=0,06\right.$;
Cuadro 2. Defectos de cierre del tubo neural, síndrome de Down y labio y paladar hendido en el Hospital Universitario de Neiva durante 1998.

\begin{tabular}{llll}
\hline $\begin{array}{l}\text { Fecha de } \\
\text { nacimiento }\end{array}$ & $\begin{array}{l}\text { Diagnóstico } \\
\text { clínico }\end{array}$ & Procedencia & Sexo \\
\hline $01 / 04 / 98$ & Down & Neiva & SI \\
$08 / 04 / 98$ & Down & Neiva & SI \\
$11 / 04 / 98$ & Nown & Neiva & SI \\
$15 / 05 / 98$ & Anencefalia & $\mathrm{F}$ \\
$22 / 05 / 98$ & Paladar hendido & Neiva & $\mathrm{F}$ \\
$05 / 06 / 98$ & Encefalocele & Campoalegre & $\mathrm{F}$ \\
$16 / 06 / 98$ & Anencefalia & Neiva & $\mathrm{F}$ \\
$10 / 07 / 98$ & Meningocele & Neiva & $\mathrm{F}$ \\
$14 / 07 / 98$ & Anencefalia & Neiva & $\mathrm{F}$ \\
$18 / 09 / 98$ & Down & Pitalito & $\mathrm{F}$ \\
$02 / 10 / 98$ & Labio hendido & Neiva & $\mathrm{M}$ \\
$08 / 10 / 98$ & Mielomeningocele & Neiva & $\mathrm{M}$ \\
$08 / 10 / 98$ & Paladar hendido & Aipe & $\mathrm{F}$ \\
$16 / 11 / 98$ & Down & Suaza & $\mathrm{M}$ \\
$26 / 11 / 98$ & Anencefalia & Campoalegre & $\mathrm{F}$ \\
$03 / 12 / 98$ & Down & Neiva & $\mathrm{F}$ \\
$30 / 12 / 98$ & Meningocele & Neiva & $\mathrm{F}$ \\
\hline
\end{tabular}

SI: sin información

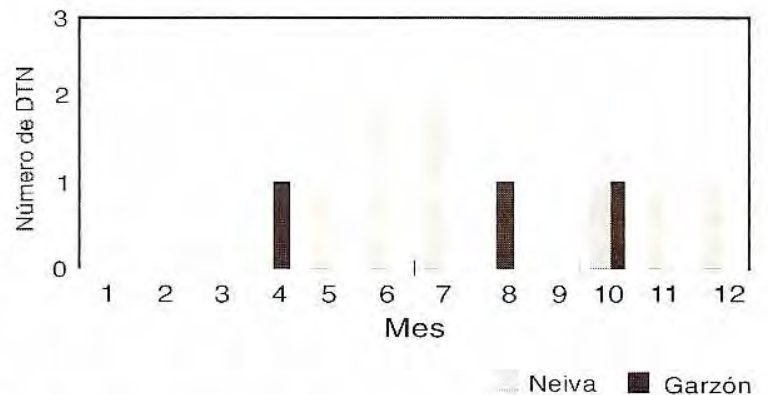

Figura 1. Distribución de los DTN por mes en Neiva y Garzón. 
Cuadro 3. Defectos de cierre del tubo neural, sindrome de Down y labio y paladar hendido en el Hospital de Garzón durante 1998.

\begin{tabular}{llcr}
\hline $\begin{array}{l}\text { Fecha de } \\
\text { nacimiento }\end{array}$ & $\begin{array}{l}\text { Diagnóstico } \\
\text { clínico }\end{array}$ & Procedencia & Sexo \\
\hline $24 / 04 / 98$ & Anencefalia & Tarqui & $\mathrm{SI}$ \\
$13 / 10 / 98$ & Down & Agrado & $\mathrm{M}$ \\
$20 / 10 / 98$ & Anencefalia & Garzón & $\mathrm{M}$ \\
$08 / 08 / 98$ & Mielomeningocele & Garzón & $\mathrm{M}$ \\
\hline
\end{tabular}

SI: sin información

Cuadro 4. Algunos defectos congénitos en el ISS de Neiva durante 1998.

\begin{tabular}{ll}
\hline Fecha de nacimiento & Diagnóstico clínico \\
\hline $04 / 98$ & Down \\
$05 / 98$ & Mielomeningocele \\
$05 / 98$ & Down \\
$07 / 98$ & Lipomeningocele \\
$09 / 98$ & Labio hendido \\
$12 / 98$ & Mielomeningocele \\
\hline
\end{tabular}

$p=0,807)$. Con relación a la tasa observada durante el período de 1990 a 1994, tampoco hay diferencia significativa $\left(\chi^{2}=1,61 ; p=0,204\right)$. La prueba de probabilidad de Poisson para eventos raros, utilizada para analizar la tasa observada de DTN en 1998 en comparación con la tasa esperada de acuerdo al promedio de los años anteriores, dio como significativo el valor de $p=0,016$.

Se observa una variación similar respecto a la razón de mortinatos; la diferencia entre 1998 y 1997 es significativa $\left(\chi^{2}=178 ; p=0,0\right)$ y también lo es comparada con la de $1996\left(\chi^{2}=70,6 ; p=0,0\right)$, pero, con relación a la razón del período de 1990 a 1994, la diferencia no es significativa $\left(\chi^{2}=1,64\right.$; $p=0,2)$. La tasa de síndrome de Down permanece estable a lo largo de los años (figura 2) y, respecto a la tasa de Latinoamérica, la diferencia no es significativa $\left(\chi^{2}=0,4 ; p=0,526\right)$.

En la figura 2 se observan las diferencias en las tasas de DTN de 1998 con los años anteriores. La línea que representa la incidencia del síndrome de Down permanece estable mientras que, a partir de 1996, se incrementan los DTN y los mortinatos; el máximo aparece en 1997 y ambos descienden en 1998.

Puesto que los hospitales de nivel I remiten los embarazos complicados al Hospital Universitario, los registros de Palermo y Baraya son nulos para abortos, mortinatos y malformados. El sesgo ocasionado por la remisión hace parecer que el problema no estuviera presente en las poblaciones vecinas o que existiera un subregistro. Sin embargo, la procedencia de los casos de DTN del Hospital Universitario indica que el $29,4 \%$ es del área rural. En el Hospital de Garzón, a 3 horas de Neiva por carretera, la infraestructura es adecuada para atender partos complicados y embarazos de alto riesgo. La tasa de los DTN no difiere en forma significativa de la del Hospital Universitario de Neiva $\left(\chi^{2}=0,39 ; p=0,534\right)$, lo mismo que la proporción de abortos $\left(\chi^{2}=1,97 ; p=0,16\right)$ y la razón de mortinatos $\left(\chi^{2}=3,68 ; p=0,055\right)$.

Esto señala que, en la actualidad, los DTN son un problema que se extiende a otras regiones de Huila donde hay factores de riesgo que no ocurren en Neiva o que son de diferente magnitud. Por ejemplo, las modificaciones sociales, culturales y ambientales de municipios cuya primordial actividad es agrícola y con alta exposición a agentes químicos de uso agrícola.

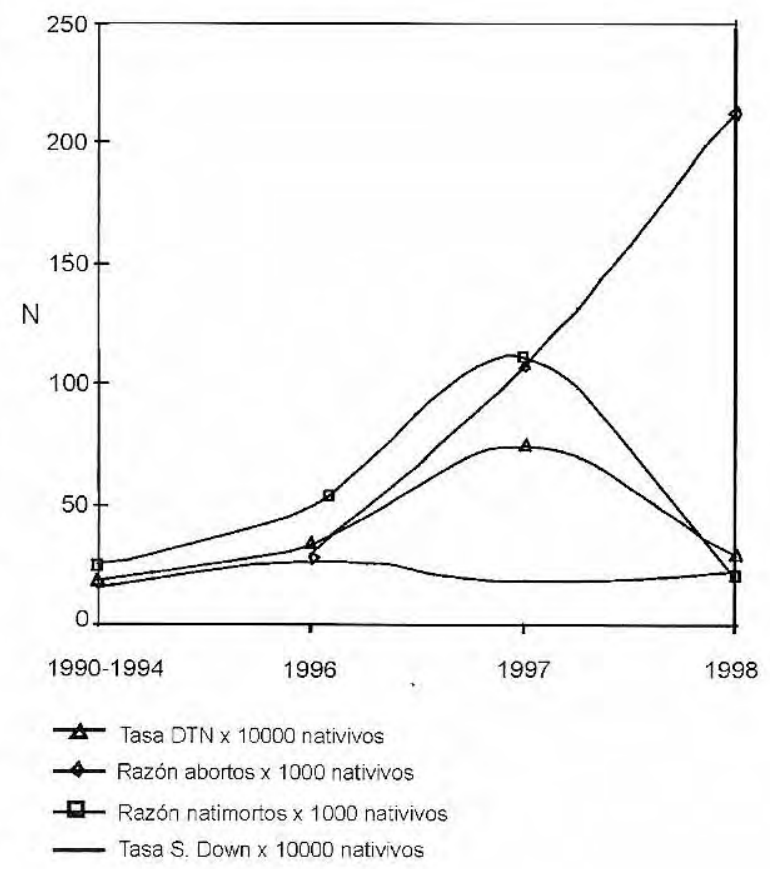

Figura 2. Tendencia de DTN comparada con síndrome de Down, aborto y natimorto. 
Cuadro 5. Mortalidad perinatal, casos y tasas de defectos congénitos seleccionados por hospital y por municipio, 1998.

\begin{tabular}{|c|c|c|c|c|c|c|c|c|c|c|}
\hline & $\begin{array}{l}00 \\
\sum^{0} \\
i n \\
00 \\
\frac{0}{0} \\
\frac{0}{20} \\
z\end{array}$ & 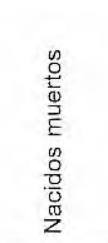 & 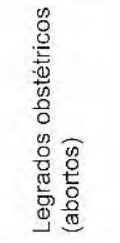 & $\begin{array}{l}\text { o } \\
\frac{0}{\pi} \\
\frac{0}{0} \\
0 \\
\frac{0}{0} \\
\frac{\pi}{0} \\
\frac{0}{0}\end{array}$ & 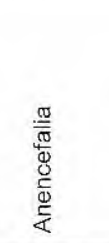 & 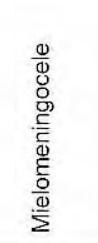 & 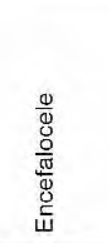 & $\begin{array}{l}z \\
\frac{z}{0} \\
\frac{\pi}{0} \\
\circ\end{array}$ & 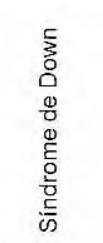 & 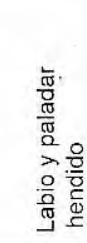 \\
\hline & $\mathrm{n}$ & Razón* & Razón* & & Tasa $^{\star *}$ & Tasa $^{* *}$ & Tasa $^{* \star}$ & Tasa $^{* \star}$ & Tasa** & Tasa $^{* \star}$ \\
\hline Palermo & 209 & - & 28,71 & 209 & - & - & - & - & - & - \\
\hline Baraya & 103 & - & 135,92 & 103 & - & - & - & - & - & - \\
\hline Garzón & 1.898 & 28,16 & 195,08 & 1.953 & 10,54 & 5,27 & - & 15,81 & 5,27 & \\
\hline ISS & 2.206 & 9,87 & 139,59 & 2.228 & 4,53 & 13,60 & - & 18,13 & 9,07 & 4,53 \\
\hline Neiva & 2.728 & 19,79 & 212,44 & 2.782 & 14,66 & 11,00 & 3,67 & 29,33 & 21,99 & 11,00 \\
\hline Latinoamérica (14) & $2 ' 546.079$ & 18,88 & SI & $2^{\prime} 595.064$ & 6,82 & 7,24 & 1,83 & 15,90 & 15,40 & 14,30 \\
\hline
\end{tabular}

SI: sin información * Razón/1.000* Tasa/10.000

Cuadro 6. Tasa de defectos del tubo neural en el Hospital Universitario de Neiva por año.

\begin{tabular}{|c|c|c|c|c|c|c|c|}
\hline \multirow[t]{2}{*}{ Período } & \multirow[t]{2}{*}{$\mathrm{RN}$} & \multicolumn{2}{|c|}{ DTN } & \multicolumn{2}{|c|}{ Abortos } & \multicolumn{2}{|c|}{ Mortinatos } \\
\hline & & $n$ & Tasa* & $n$ & Razón** & $n$ & Razón ${ }^{* *}$ \\
\hline $1990-94(15)$ & 15.254 & 28 & 18,0 & $\mathrm{SI}$ & SI & 363 & 23,8 \\
\hline 1996 & 3.039 & 10 & 32,9 & 86 & 28,3 & 150 & 49,0 \\
\hline 1997 & 2.291 & 17 & 74,2 & 245 & 106,9 & 253 & 110,4 \\
\hline 1998 & 2.728 & 8 & 29,3 & 591 & 212,4 & 54 & 19,8 \\
\hline
\end{tabular}

RN: recién nacidos vivos

SI:sin información
* Tasa/10.000
* Razón/1.000

Como punto de comparación para la maternidad del Hospital Universitario, se buscó la información de la Clínica del Seguro Social en Neiva. El sistema de registro es diferente, pues no está diseñado para registrar anomalías congénitas; por tanto, sólo se pueden contabilizar los casos severos que han tenido necesidad de ser internados en la unidad de cuidados intensivos. Por ello, se considera que hay subregistro en esta clínica y las tasas no son útiles para comparación, pero señalan que los DTN también se presentan en otras maternidades de la ciudad.

La proporción de abortos o legrados obstétricos ha tenido un incremento en los últimos dos años. La explicación más razonable que se le pudo asignar a este hecho es que en los últimos años el registro de aborto se ha modificado para incluir todos los legrados que llegan para ser atendidos en la maternidad; por tanto, su tendencia al aumento puede ser un sesgo de selección del registro actual y no un aumento real.

La explicación para la caída en la tasa de DTN no puede ser solamente la fortificación de la harina de trigo, ni el hecho de haber implementado acciones para el consumo de alimentos ricos en ácido fólico, iniciada por la Secretaría de Salud del Huila desde finales de 1997. En esa época había mujeres embarazadas que tuvieron su hijo en 1998 y para entonces ya había ocurrido el cierre del tubo neural en el embrión, o su defecto, sin que ello pudiera modificarse por las medidas tomadas. Es posible que la divulgación y motivación del personal médico y paramédico y su insistencia en el consumo de ácido fólico preconcepcional, iniciada desde 1996, también haya contribuido. Esta es una medida que, en la actualidad, se acepta como parte de la estrategia de mejorar el consumo de ácido fólico (16). 
El principal punto de discusión está en que no se han monitorizado factores ambientales de riesgo, que se han sugerido como importantes, tratándose de una región agrícola con elevada aspersión con plaguicidas. Esta posible influencia se informó después de evaluar los casos de 1990 a 1994 , pero aún no se ha descartado ni demostrado mediante algún método de laboratorio (10).

Hasta el momento, los resultados indican que hay una reducción real en los DTN en el último año en Neiva y es posible que las medidas de promoción del consumo de ácido fólico en la dieta y la suplementación médica hayan influido. Para aceptar que la disminución en la tasa de DTN sea un indicador del impacto de la fortificación de harinas, se necesita evaluar los otros factores de riesgo y, para ello, se deberá establecer un protocolo de vigilancia.

\section{Conclusión}

Los DTN disminuyeron en el Hospital Universitario de Neiva en forma significativa en 1998. Aunque no se puede definir la razón de esta disminución, se sospecha que influyeron las medidas de intervención para la suplementación iniciadas con el personal del Hospital en 1996 y continuadas en 1997 con campañas de divulgación sobre el consumo de ácido fólico y la adopción de la norma sobre fortificación de harinas. Esto se deberá corroborar mediante el establecimiento de un protocolo de vigilancia.

Resaltamos la utilidad de la estrategia de insistir entre el personal médico y paramédico sobre la formulación de ácido fólico a las mujeres en edad fertil, pues la mayoría de mujeres consultan para el control del embarazo cuando éste va en curso y el tubo neural ya se ha cerrado o ha quedado con defecto.

Aunque no se desprende de la información de este estudio, según la experiencia informada en otros países, es claro que se deben estudiar las actitudes y prácticas de la mujer en esta región con respecto a las recomendaciones del consumo de folatos, lo cual permitirá saber cómo llegar a la comunidad.

\section{Referencias}

1. Burke B. Preventing neural tube defects. Atlanta: Centers for Disease Control and Prevention; 1998.
2. Hall JG, Friedman JM, Kenna BA, Popkin J, Jawanda M, Arnold W. Clinical, genetic and epidemiological factors in neural tube defects. Am J Hum Genet 1988;43: 827-37.

3. Canfield MA, Annegers JF, Brender JD, Cooper SP, Greenberg F. Hispanic origin and neural tube defects in Houston/Harris County, Texas. II. Risk factors. Am J Epidemiol 1996;143:12-24.

4. Ministerio de Salud, República de Colombia. Decreto 1.944 de octubre de 1996.

5. Division of Media Relations, Centers for Disease Control and Prevention. What CDC is doing to prevent spina bifida and other neural tube defects (NTDs). MMWR Morb Mort Wkly Rep 1996:1-4.

6. Teratology Society. Consensus statement on the use of folic acid to reduce the risk of birth defects. Teratology 1997;55:381.

7. Oakley GP. Folic acid-preventable spina bifida and anencephaly. JAMA 1993;269:1292-3.

8. van der Put NM, Gabreels F, Stevens E, Smeitink JAM, Trijbels F, Eskes $\mathrm{T}$, et al. A second common mutation in the methylenetetrahydrofolate reductase gene: an additional risk factor for neural tube defects? Am J Hum Genet 1998;62:1044-51.

9. Abramszky L, Botting B, Chapple J, Stone D. Has advice on periconceptional folate supplementation reduced neural-tube defects? Lancet 1999;354:998-9.

10. Ministerio de Salud-INS. Incremento en los defectos del tubo neural en nacidos vivos en el Hospital General de Neiva. Inf Quinc Epidem Nac 1997;2:330-2.

11. Thompson M, Mclnnes R, Willard H. Genetics in medicine. Fifth edition. London: W.B. Saunders; 1991. p.355.

12. Centers for Disease Control and Prevention. Knowledge and use of folic acid by women of childbearing age, United States, 1997. MMWR Morb Mort Wkly Rep 1997;46:721-3.

13. Secretaría de Salud Departamental, Gobernación del Huila, República de Colombia. Resolución No.0176 de 1999.

14. Estudio Colaborativo Latinoamericano de Malformaciones Congénitas, ECLAMC. Informe solicitado sobre defectos de DTN en Latinoamérica para 1998. Instituto Fiocruz, Brasil; 1998.

15. Ostos H, Bermúdez A, Sánchez C. Frecuencia de defectos de tubo neural en la población de Neiva, Colombia: estudio de seguimiento con base hospitalaria 1990-94. Biomédica 1997;17(Supl.1):94-5.

16. Division of Media Relations, Centers for Disease Control and Prevention. Los CDC piden instruir mejor al personal médico para fomentar el consumo diario de ácido fólico en las mujeres. Atlanta: CDC 1999. p.1-2. 